\title{
Culturas políticas del nacionalismo español. El caso de la ciudad de Valencia (1958-1979)
}

\author{
JuAn Carlos Colomer Rubio \\ Universitat de València (Espanya) \\ orcid.org/0000-0002-4677-201X
}

Presentació: 30 set. 2014 | Acceptació: 31 gen. 2015 | Publicació: 15 des. 2015

Citació recomanada: Colomer Rubıo, Juan Carlos. Culturas políticas del nacionalismo español. El caso de la ciudad de Valencia (1958-1979). Franquisme \& Transició. Revista d'Història i de Cultura 3 (2015): 157-172. doi: 10.7238/fit.voi3.2402.

Resumen: El estudio de las grandes culturas políticas del franquismo se ha detenido en el análisis de ciertos discursos y prácticas que emanaban de los grandes líderes políticos del régimen. Desde nuestro punto de vista, ha faltado una necesaria reflexión sobre la concreción del desarrollo de esas culturas políticas en el régimen local. El aumento, en número y profundidad, de los estudios sobre la evolución política e ideológica de las instituciones locales en el régimen, unido a un creciente número de los centros documentales que lo han permitido, ha posibilitado avanzar en dicho conocimiento pero sin la consiguiente complementación con un estudio de las culturas políticas del nacionalismo español en lo local. Por tanto, reduciendo la escala de observación y concretando dicho discurso, hemos optado por la selección y análisis de las trayectorias de los principales líderes políticos del Ayuntamiento de Valencia atendiendo a la fase histórica 1958-1979, donde, a nuestro parecer, podemos detectar un cambio y transformación de las dos culturas políticas del régimen: la fascista y la nacionalcatólica. Ambas, con una misma idea de nación pero con diferentes formas de interpretarla, tendrán en la localidad su máximo cauce de expresión, en tanto en cuanto espacio de múltiples relaciones y discursos diversos, así como espacio de transferencia entre ambas identidades en conflicto y relación permanente. Palabras clave: Culturas políticas, franquismo, Valencia, fascismo, regionalismo

Este artículo se ha redactado en el contexto del proyecto Derechas y nación en la España contemporánea. Culturas e identidades en conflicto (HAR2014-53042-P) financiado por la Dirección General de Investigación Científica y Técnica del Ministerio de Economía y Competitividad. 


\section{Political cultures of Spanish nationalism. The case of the city of Valencia (1958-1979)}

Abstract: The study of the major political cultures of Franco was arrested in the analysis of certain discourses and practices emanating from the great political leaders of the regime. From our point of view, it has lacked a necessary reflection on the realization of the development of these political cultures in the local regime. The increase in number and depth of the literature on the political and ideological evolution of local institutions in the system, coupled with a growing number of documentation centres that have permitted, has enabled progress in such knowledge but subsequent complementation to a study of political cultures of Spanish nationalism in the local. Therefore, reducing the scale of observation and specifying that speech, we have opted for the selection and analysis of the trajectories of the main political leaders of the City of Valencia in response to the historical period 1958-1979, which, in our view, we can detect a change and transformation of the two political cultures of the regime: fascist and national catholic. Both with the same idea of nation but different ways of interpreting it, have locally maximum channel of expression, as long as space of multiple relationships and various speeches. Keywords: Political Cultures, Franco, Valencia, fascism, regionalism

En los últimos años el aumento, en número y profundidad, de los estudios sobre la evolución política e ideológica de las instituciones locales en el régimen franquista, unido a una creciente extensión del número de archivos que lo han permitido, ha posibilitado avanzar en un conocimiento amplio sobre la instauración y evolución del régimen pero sin la consiguiente complementación con un estudio de las culturas políticas del franquismo español, especialmente en el ámbito local. ${ }^{1}$ El estudio de las grandes culturas políticas del franquismo se ha detenido en el análisis de ciertos discursos y prácticas que emanaban de los grandes líderes

1 El presente trabajo forma parte de una tesis doctoral sobre las elites locales valencianas entre el franquismo y la democracia atendiendo especialmente a las que desarrollaron su actividad política en el Ayuntamiento de la ciudad. La tesis, de pronta publicación, fue defendida por el autor en julio de 2014. Véase: Juan Carlos Colomer, Gobernar la ciudad. Alcaldes y poder local en Valencia, 1958-1969 (tesis doctoral, Universidad de Valencia, 2014). 
políticos del régimen. Desde nuestro punto de vista, ha faltado una necesaria reflexión sobre la concreción del desarrollo de esas culturas políticas del nacionalismo español en el plano del régimen local. ${ }^{2}$

Por tanto, reduciendo la escala de observación y concretando dicho discurso, hemos optado por la selección y análisis de las trayectorias de los principales líderes políticos del Ayuntamiento de Valencia atendiendo a la fase histórica 1958-1979, donde, a nuestro parecer, podemos detectar cierta transformación de las dos culturas políticas del régimen: la fascista y la nacionalcatólica. ${ }^{3}$ Ambas, con una misma idea de nación pero con diferentes formas de interpretarla, tuvieron en la localidad su máximo cauce de expresión, en tanto en cuanto espacio de múltiples relaciones y discursos diversos, así como espacio donde se dieron las necesarias transferencias entre ambas identidades en conflicto y relación permanente. ${ }^{4}$

Ahora insistimos en que lo nacional no se limitó a eliminar o a sobreescribir la identidad local. En vez de ello, destacamos cómo lo local se apropia de lo nacional, cómo la nación adquiere diferentes significados locales, cómo lo local es celebrado en el pensamiento nacionalista como el hogar de la nación, y cómo la nación pretende ser lo realmente local. Y recordamos que ninguna identidad nacional moderna ha podido ser operativa sin dejar de ignorar lo local y sin elaborar sus propias concepciones acerca de lo local y de la identidad regional..$^{5}$

2 Algunas excepciones reseñables pueden encontrarse en: Antonio Canales, Las otras derechas: derechas y poder local en el País Vasco y Cataluña en el siglo XX (Madrid: Marcial Pons, 2006); Claudio Hernández, Granada Azul. La construcción de la «Cultura de la Victoria» en el primer franquismo, (Granada: Comares, 2011).

3 Aceptamos, como punto de partida, la división propuesta por Ismael Saz y desarrollada en varios de sus trabajos. Destacamos aquí: Ismael SAZ, «Las culturas de los nacionalismos franquistas», Ayer 71 (2008): 153-174, y del mismo autor: «La historia de las culturas políticas en España (y el extraño caso del "nacionalismo español")», en Benoit Pellistrandi \& Jean-François Sirinelli, L'histoire culturelle en France et en Espagne (Madrid: Casa Velázquez, 2008), 215-234.

4 Una misma preocupación puede encontrarse en el desarrollo efectuado por Alain Confino. Véase: Alain Confino, «Lo local, una esencia de toda la nación», Ayer 64 (2006):19-31. Para el caso español debemos destacar el trabajo realizado por Claudio Hernández para el caso de Granada. Una síntesis puede leerse en Claudio HERNÁNDEZ, “"Desempolvando las camisas”: revitalización falangista y combate por España en el marco local», en Miguel Ángel Ruiz, ed., Falange. Las culturas políticas del fascismo en la España de Franco (1936-1975) (Zaragoza: Institución Fernando el Católico, 2013), 221-239.

5 Alain Confino, «Lo local, una esencia de toda la nación», 22. 
Con ello partimos del ámbito local para detectar si esos debates mantenidos por ambas culturas políticas en esferas superiores tenían su transferencia en las localidades, además de escapar de la tradicional cronología, de 1948-1953, que ha dominado hasta la actualidad estos estudios. El mal considerado segundo franquismo puede aportar muchas claves sobre el mantenimiento del debate discursivo mantenido por ambas culturas políticas del régimen, pero también su progresiva transformación a lo largo del tiempo.

Así, a una necesaria introducción historiográfica que sirva de planteamiento de partida, realizaremos el análisis de las ideas de nación concretadas en el plano local por parte de los líderes que ocuparon los principales puestos de poder, en especial en el Ayuntamiento de Valencia. A un ideal fascista de nación puesto en práctica durante la alcaldía de Adolfo Rincón de Arellano, de 1958 a 1969, pasaremos a una concreción del concepto de nación complejo y diverso basado en una identidad pluriregional, sustentado en la región como entidad administrativa, y que defendieron los últimos líderes locales franquistas valencianos. La derivación posterior de éstos hacia un regionalismo político, diferente a los planteamientos de nación propugnados por el fascismo, ocupará parte de nuestra reflexión. Ello nos permitirá concluir con la pluralidad y diversidad de formas en la que se concretaron las culturas políticas de los nacionalismos franquistas en el plano local y que rompe con el modelo de independencia entre ambas, enfatiza las conexiones y transferencias, así como su evolución posterior al final del régimen. El caso de la ciudad de Valencia es buena prueba de ello.

\section{Las culturas políticas en la España de Franco}

¿Es posible hablar de culturas políticas dentro de la España de Franco? ¿Cómo delimitarlas?

En primer lugar, debemos destacar que el avanzado estado de la investigación sobre el franquismo ya nos permite superar el concepto monolítico de Almond y Verba sobre cultura política y nos abre la puerta a hablar de una diversidad de culturas políticas nacionales dentro de 
una misma nación. ${ }^{6}$ Varias formas de ver, entender y vivir la nación compartidas por la misma cultura política. Así, en la actualidad asistimos a una superación del concepto propugnado por ambos autores, combinado con un eclecticismo de la definición de Baker con la de Sirinelli, afirmando que: «En particular, consideramos que una cultura política es, en efecto, un conjunto de discursos, o prácticas simbólicas» que se complementa con «un conjunto de representaciones que configura un grupo humano en el plano político, es decir, una visión del mundo compartida, una común lectura del pasado, una proyección en el futuro vivida conjuntamente»?

Por tanto, es evidente que podemos detectar una vivencia compartida de nación y su representación que transita al plano local y que es propugnado por ciertos líderes locales. Por otro lado, ese mismo ejemplo local nos da resultados diversos dado que en la localidad los límites de las culturas políticas nacionales van a resultar más imprecisos y difusos.

Deteniendo nuestra mirada a las culturas políticas de la España de Franco con una idea de nación determinada vemos que, para el caso de la cultura política fascista, la idea de nación se situaría por encima de partidos e ideologías y, con una clara vocación imperial, admitía unos fuertes límites y no daba pie a quiebras o grietas. Era, por tanto, una nación eterna, «unidad de destino en lo universal, patria común e indivisible». Por otro lado, por lo que respecta a la nación nacionalcatólica, poseía los límites internos marcados precisamente por las instituciones y elites económicas, sociales, militares, eclesiásticas a las que apelaba, y los externos. Era elitista y tendía a anteponer la administración a la política. La participación popular en la vida política, por más controlada que estuviera, contendría potenciales desarrollos democráticos y liberales. Planteaba, incluso, el potencial de la región como constructo de nación, una idea que podrá ser detectada claramente en el plano local. ${ }^{8}$ Como ha desta-

6 Gabriel Almond y Sidney Verba, «La cultura política», en Albert Batlle, ed., Diez textos básicos de ciencia política (Barcelona: Ariel, 1992), 171-201.

7 Ismael SAZ, «Las culturas políticas del nacionalismo español», en Manuel Pérez Ledesma \& María SIER RA, Culturas políticas: teoría e historia (Zaragoza: Institución Fernando el Católico, 2010), 328.

8 Ismael SAZ, «Fascismo y nación en el régimen de Franco. Peripecias de una cultura política», en Ruiz, ed., Falange, 68. Una buena aproximación de esta defensa de la identidad puede leerse en 
cado A. Geniola, el franquismo utilizará la región con fines diversos. En un primer momento, hasta bien entrados los años sesenta, primó destacar el aspecto regional como fundamental componente de la tradición histórica española pero despojado de cualquier reivindicación política. La crisis del régimen, y la posterior transición, enfatizó otros elementos propios de la región, más funcionales y políticos, con cierto peso autonomista. Ello se verá en la reivindicación de los principales líderes locales valencianos. ${ }^{9}$

Como vemos, la existencia de dos ideas de nación vinculadas a una explotación de la región dentro del franquismo y cuya continuidad es mayor a lo comúnmente subrayado resultó una constante en la dictadura. Queda, por tanto, analizar y ver qué concreción local pudieron tener ambos discursos y qué desarrollo plantearon las elites locales a dichas construcciones discursivas presentes en las esferas generales del régimen. Ello permitirá responder a dos cuestiones fundamentales: ¿hasta qué punto los discursos generales son homogéneos o permeables? ¿Qué idea de nación podían tener los mandatarios, en especial los líderes municipales, en constante relación con los intereses de los administrados y la realidad provincial que gobernaban y vivían?

\section{Valencia e identidad nacional fascista (1958-1969)}

La selección de un espacio local como la ciudad de Valencia no es una cuestión baladí por una serie de circunstancias. En primer lugar, la ciudad, que contaba con 505.066 habitantes a la altura de 1958, vivió una serie de transformaciones socioeconómicas muy importantes derivadas de dos hechos fundamentales: el desarrollo turístico de toda el área mediterránea en ese contexto y un desastre natural, la riada del rio Turia a su paso por la ciudad, en 1957, que dejó una ciudad devastada y afectada en su trama urbana lo que obligó a una política de reconstrucción severa. Además, en segundo lugar, el peso del sector monárquico de la ciudad,

Sara PRADES, «Escribir la historia para definir la nación: la historia de España en Arbor, 1944-1956», Ayer 66 (2007): 177-200.

9 Andrea Geniola, «"Es tan sano el regionalismo valenciano”. Regionalisme i anticatalanisme al País Valencià durant el franquisme (1962-1977)», Afers 79 (2014): 619-641. 
encabezado por su alcalde, el aristócrata Tomás Trénor Azcárraga, llevó a duras críticas públicas por la gestión del desastre y a una crisis política sin precedentes sobre la cual ya nos hemos ocupado en otra ocasión. ${ }^{10}$ El peso político de la ciudad, unido a las circunstancias anteriores, llevó al régimen político franquista a reconducir políticamente a la urbe hacía unos presupuestos políticos diferentes a los que la habían gobernado anteriormente a la riada. Para ello, optaron por un alcalde falangista, camisa vieja, con ideas divergentes y un estilo de gobierno distinto al de mandatarios anteriores. La persona elegida era Adolfo Rincón de Arellano. ${ }^{11}$ Su postura ideológica, explicitada personalmente en una publicación años después, ya marcaba claramente la diferencia con alcaldes anteriores y propugnaba los ideales populistas y antiseparatistas defendidos por la cultura política falangista:

Mi inconformismo ante las injusticias sociales que el Estado liberal con su indiferentismo era incapaz de resolver y mi oposición al sistema capitalista, mi desprecio por la política al uso, que provocaba cambios de Gobierno sin haberles dado tiempo de desarrollar ninguna labor constructiva. Mi repugnancia ante el servilismo de nuestros Gobiernos ante los deseos de las potencias extranjeras, y de lástima ante el papanatismo de muchos españoles que creían que siempre lo de fuera es mejor. Mi repulsa ante la estúpida destrucción de riquezas y obras de arte, que en todo caso podrían haber llegado a ser patrimonio de todos. Mi preocupación ante los brotes de separatismos, política antirreligiosa, desorden público, huelgas, anarquía en el campo: destrucción de cosechas, árboles, ganados... Asesinatos. Impunidad... que trabajaban en beneficio del imperialismo soviético. Todos estos hechos trajeron como consecuencia nuestra guerra civil. Guerra civil que José Antonio trató de evitar con la creación de Falange, que buscaba sobre todo la unidad de los espa-

10 Juan Carlos Colomer, «"Gobernar la ciudad”. El Ayuntamiento de Valencia de la dictadura a la democracia: un estudio de caso (1969-1979)», Hispania 245 (2013): 257-272.

11 Adolfo Rincón de Arellano constituye un personaje fundamental de la elite local valenciana del franquismo. Proveniente del mundo jonsista, luchó en la Guerra Civil y ocupó la primera secretaría provincial del Movimiento acabada la guerra. Sus contactos políticos con Madrid le llevaron a una carrera política meteórica que culminó en la alcaldía de Valencia hasta su dimisión en 1969, año de la crisis del proyecto falangista. 
ñoles. Habló pero no fue escuchado y la Falange que había nacido para unir a los españoles, tuvo que ser beligerante en una guerra entre hermanos. ${ }^{12}$

Esa beligerancia ante el separatismo y esa propuesta de nación homogénea, defendida desde el consistorio presidido por Rincón de Arellano, no sólo se manifestará en discursos políticos o actitudes personales concretas, sino en las políticas públicas puestas en marcha por el Ayuntamiento como, por ejemplo, el aumento de los elementos y símbolos del nacionalismo español presentes en festividades públicas como las fallas y que serán una constante en todo el periodo. ${ }^{13}$ La más recordada será la quema de una figura alegórica del escritor Joan Fuster en las fallas de 1963 y que será aplaudida por el Ayuntamiento, en tanto en cuanto ataque a un «antiespañol», o la beligerancia que el propio consistorio tendrá hacia el uso del valenciano dentro del espacio social de la ciudad y el ostracismo al que lo condenará. ${ }^{14}$ Todos estos elementos no son menores pues constituyen elementos fundamentales del «nacionalismo banal» español que, impulsado de forma indirecta, conectaba claramente con sectores sociales amplios que podían sentirse identificados con una cultura política concreta. ${ }^{15}$

El refuerzo de la identidad nacional española, impulsado por el falangismo presente en el consistorio municipal valenciano, especialmente desde 1958, no invalidará vías intermedias que conectaban con la otra cultura política nacionalista, especialmente en lo que al uso del regionalismo se refiere. Aquí, es muy importante el uso del factor folclórico, que

12 Miguel Veyrat \& José Luis Navas-Migueloa, Falange, hoy (Madrid: G. del Toro Editor, 1973), 261.

13 Otras comisiones falleras también realizaron el mismo escarnio de la figura de Fuster. La de la comisión Cádiz-Literato Azorín, con un grupo crítico dedicado a Nosaltres, els valencians, veía las tesis de Fuster como «influencias extranjeras en nuestra costumbre». Y en el monumento de ese año, la Falla de la plaça de la Mercè quemó un ninot del intelectual rodeado de versos críticos como estos: «Mostra ser pantomimer | i per la seua expressió | mereix de fet l'expulsió | d'este país tan sincer. || Que es menge el pa d'altre lloc; | que ací a València volem | fills que no ens tiren al fem | com ens ha llençat fa poc». Un buen trabajo sobre franquismo y mundo fallero puede leerse en Gil Manuel HERNÁNDEZ, Falles i franquisme a València (Valencia: Afers, 1997).

14 El caso más destacable será la utilización de la lengua castellana como única lengua vehicular de la fiesta fallera en actos oficiales. La designación como responsable de las fallas de Juan Bautista Martí Belda, figura de relevancia local en la fiesta, no hizo más que enfatizar esta realidad; cf. Levante, 5 nov. 1963 .

15 Michael BiLlig, Nacionalisme banal (Catarroja: Afers, 2006). 
no político, de la región. La región se utilizará sólo ahora como una realidad histórica, no política, y cuya razón de ser es simplemente por su contribución a las esencias de la nación española. Así, en la visita de Franco a la ciudad en 1962, el propio Rincón de Arellano se refería a Valencia en estos términos:

Excelencia: Valencia está aquí, abierta y ardiente, como su cielo, como su amanecer. ¿La veis? Es esa que os aplaude y espera. Su voz y su grito. Toda la ciudad, y con ella la región en vilo, cuajada y como solidificada en mi voz, os habla. [...] una región miembro vivo de un cuerpo nacional en plena vitalidad, la de la gratitud sin mohos de resabios ni ficciones, por ti y contigo, en adhesión de fe y amor, es la que te ofrecemos, es la que está contemplando: Valencia en tu presencia. ${ }^{16}$

Por tanto, la región, desde un plano local y desde un tinte folclórico, comenzará a implementarse desde dos vías complementarias: la utilización de la fiesta fallera y el uso interesado de elementos y signos de la región sin significancia política. Dicha integración del elemento regional como parte básica de la «nación inmortal» por parte de miembros de la cultura política fascista nos hablará claramente de la integración y variación del ideal de nación de una cultura política derivado de su contacto con la realidad local.

Este énfasis de la región que va a realizar cierto sector falangista y que modificará ciertos preceptos de su cultura política, conectaba con el sentimiento regionalista valenciano de preguerra y que es indisociable a la realidad local analizada. De hecho, en este sentido, debemos tener presente que las primeras identidades colectivas contemporáneas de los valencianos eran prácticamente, de una manera coetánea, la identidad nacional española y la identidad regional valenciana, algo común en el resto de la Península. Ambas identidades, lejos de ser incompatibles, se articularon de manera jerárquica. De hecho, la identidad valenciana se produjo dentro del nacionalismo español, como vemos en el ejemplo siguiente:

16 Discurso de Rincón de Arellano en la visita de Franco a la ciudad en 1962 extraído del diario Pueblo del 18 de junio de 1962. 
Valencia cuenta con generales simpatías. No se nos puede regatear afectos porque estamos tan orgullosos de ser valencianos como españoles, aquí no anida tibia la idea de patria, carecen en absoluto de ambiente los anhelos de disgregación, sino dejar de amar nuestra vieja Senyera, jamás humillada, le cedemos siempre el paso a la bandera española; por todo estos somos acreedores a que toda España contemple la gallarda expresión de nuestro progreso regional. ${ }^{17}$

El propio Serrano Suñer, absolutamente convencido del sentido patrio del regionalismo valenciano, hablaba en estos términos en 1940:

Valencia, teniendo elementos bien definidos para construir una unidad regional bien caracterizada [...], ha sido, sin embargo, y pese a todos los autonomistas que en su tierra fueron, fiel en todo momento al destino unitario de España. $^{18}$

Por tanto, el desarrollo de la cultura política del nacionalismo español propugnado por Falange y concretamente por las elites locales falangistas de la ciudad, sin distanciarse de los preceptos culturales del partido único, plantea nuevas vías regionalistas, de reivindicación de la región histórica, gracias al contacto con lo local y que serán aprovechadas por líderes de las otras culturas políticas.

\section{Entender la nación desde la región política (1973-1979)}

Si bien el peso de la cultura política nacional fascista fue entrando en clara decadencia a partir de 1969, fecha que coincidiría con la salida de Rincón de Arellano de la alcaldía de Valencia, detectamos en la ciudad un énfasis del vector regionalismo entendido ahora desde el autonomismo y el peso político de la región, que conecta claramente con lo sucedido en otras zonas peninsulares y que aún hoy ha sido escasamente estudiado. ${ }^{19}$

17 Citado por Josep Vicent BoIra, València i Barcelona. Retorn al futur(Valencia: 3i4, 2006), 84.

18 Citado por Geniola, «"Es tan sano el regionalismo valenciano”...», 624.

19 Un caso paradigmático es el de Hipólito Gómez de las Roces, último presidente de la Diputación franquista de Zaragoza y cuya derivación política posterior le llevará a la fundación del PAR 
Este nuevo regionalismo que parte de cierto folklorismo, acabará derivando en una clara opción política, lo que nos permite descubrir una nueva deriva política de las culturas políticas franquistas hacia el regionalismo. Dicha derivación ya puede detectarse en una misiva enviada por el valenciano Enrique Oltrá Moltó, gobernador civil de Guipúzcoa, al todavía alcalde de Valencia Rincón de Arellano en 1969:

Sabes que soy valenciano por los cuatro costados. Hay por aquí, por estas latitudes [Guipúzcoa] un mal entendido regionalismo que no se sabe muchas veces donde acaba y donde empiezan otras cosas. Pongo muchas veces el ejemplo del regionalismo sano y auténtico de nuestro sentir valenciano que ama entrañablemente a nuestra tierra y que, al mismo tiempo, por sentirse irrevocablemente vinculado a la misma, se sabe también irrenunciablemente español. Que la historia de Valencia y la historia de Vasconia nada serían desgajadas de la Historia de España de la que a Dios gracias formamos parte. [...] Por añadidura, los que nos sabemos valencianos sentimos también el legítimo orgullo de la íntima satisfacción de experimentar como nuestro gran amor a la Región valenciana, no sólo no supone óbice alguno para amar a la Patria grande, sino, todo lo contrario, nos empuja a ella y nos la hace amar con más intensidad. ${ }^{20}$

En dicha misiva se diferencia entre el sano regionalismo apegado necesariamente a lo español y que, si todavía no nos permite asociarlo a un constructo político independiente, podemos incluirlo como elemento interesante de avance y continuidad de determinada cultura política. Dicha continuidad se podrá ver más claramente una vez la alcaldía de Valencia fuera ocupada, a partir de 1973, por una persona que enlazará claramente con los postulados de ese regionalismo político que podemos inscribir, con matices, con la cultura nacionalcatólica. Esa persona era Miguel Ramón Izquierdo, alcalde de 1973 a 1979. ${ }^{21}$

\footnotetext{
(Partido Aragonés Regionalista) en 1978.

20 Correspondencia de Adolfo Rincón de Arellano con Gobernadores Civiles de otras Provincias, Fondo Rincón de Arellano, caja 57, Archivo del Reino de Valencia.

21 Ramón Izquierdo, valenciano de nacimiento y proveniente del sector de la abogacía, adquirió un importante prestigio en la ciudad como presidente del Colegio de Abogados. Figura de reconocido prestigio en el mundo regionalista, perteneció a una elite profesional y técnica que tuvo que
} 
Para el caso del Ayuntamiento de Valencia, y como se extrae del discurso de investidura del nuevo alcalde, se enfatizó la idea de fomentar un «regionalismo bien entendido como fórmula seria» para avanzar hacia el progreso y bienestar del pueblo valenciano. Una idea que continuó en el discurso de toma de posesión, tras las supuestas elecciones municipales de 1976, y muy presente en la ideología del propio alcalde:

En cuanto al regionalismo, destaca la conveniencia de que los valencianos afirmemos, cada vez más, una presencia definida y decisiva en el conjunto hispano [...]. El Ayuntamiento de la Capital no puede permanecer ajeno a estas inquietudes. La unión de los valencianos importa mucho para la consecución de estas legítimas aspiraciones, para que los postulados regionalistas sirvan a la autonomía y a la descentralización, y éstas a su vez a los intereses nacionales. $^{22}$

Por primera vez se apostaba por cierto grado de autonomía y descentralización desde una institución local como el Ayuntamiento. Ahora la región se reivindicaba también como constructo político, e incluso autonómico. En la base de esa evolución encontramos dos explicaciones: por un lado, la respuesta al auge de las propias ideas autonomistas especialmente después de la muerte del dictador y, por otro, que esta vía era la única forma de defender una idea de región, muy limitada políticamente, que de otra manera no hubiera tenido peso en el nuevo escenario político.

Ese giro se concretó en políticas impulsadas por la propia elite, en especial del propio alcalde, como por ejemplo la firma del documento solicitando autonomía para la región valenciana promovido por el consejero nacional del Movimiento José María Adán García. En dicho manifiesto se afirmaba:

\footnotetext{
hacer frente al proceso transicional desde las instituciones locales. Su vida estuvo marcada por su militancia política en defensa de los valores considerados por él «genuinamente valencianos». La defensa del «idioma valenciano», y de los símbolos y tradiciones «patrias» siempre en relación y acatamiento extremo de los principios del Movimiento, mientras los hubo, constituyó la característica fundamental de su mandato.

22 «Actas del pleno municipal», Archivo Miguel Ramón Izquierdo, carpeta «Discursos», D-495, Archivo Municipal de Valencia.
} 
Toda la autonomía para la región valenciana deberá descansar en el respeto profundo a su identidad histórica, su personalidad y su cultura propia, permitiendo su pleno desarrollo sin interferencias ni dependencias de ningún género. A tal fin y por ser Valencia una realidad armónica y bilingüística, los idiomas oficiales serán el valenciano y el castellano. La región valenciana quedará abierta a todas las fórmulas de colaboración, no sólo con el Estado Nacional, del que es parte integral, sino de todas las regiones, sin preferencias ni discriminaciones con respecto a ninguna de ellas. ${ }^{23}$

Además, el año clave será 1977 en el contexto de celebración de las primeras elecciones generales de la democracia. Ese momento de fuertes expectativas políticas motivó una declaración anticatalanista y regionalista del consistorio afirmándose en los términos siguientes del regionalismo ya señalado:

El Ayuntamiento en la sesión plenaria celebrada ayer, aprobó por unanimidad la propuesta formulada por la comisión de cultura como resultado de la moción presentada en la anterior sesión plenaria por el señor Pascual Lainosa con referencia a la personalidad valencia y el término 'Paisos Catalans' [sic]. Dicha propuesta contiene los puntos siguientes: $1^{\circ}$ Reafirmar la personalidad propia histórica y actual de la región valenciana cuyas razones justificativas por obvias y evidentes se considera innecesario manifestar. $2^{\circ}$ Expresar que, consecuentemente y sin perjuicio de la superior unidad de los hombres y las tierras de España, la región valenciana es totalmente independiente de cualquiera otras, oponiéndose este excelentísimo Ayuntamiento a que se integre en la artificial denominación de 'Paisos Catalans'. $3^{\circ}$ Por ende, este excelentísimo Ayuntamiento considera que, en cualquier cuestión o asunto, la región valenciana no puede ni debe estar representada más que por sus propios mandatarios y no por los de otra región cualquiera que sea el título en el que esta pretenda basarse para ello. $4^{\circ}$ Dar cuenta al Rey y al presidente del Gobierno de los acuerdos que anteceden. ${ }^{24}$

23 José María AdÁn García, Al servicio de Valencia: apuntes para la historia, 1967-1978 (Valencia: Fórum de la concordia, 2005), 65-66.

24 «Actas del pleno municipal del 4 de marzo de 1977», D-498, Archivo Municipal de Valencia; Diario Las Provincias, 5 de marzo de 1977. 
Esa declaración no sólo era mera posición ideológica sino que era la expresión directa y concreta del sentir del consistorio en este tema. El alcalde, en plena conexión con el concejal responsable de la fiesta fallera, impulsaba dicho escrito como expresión clara de su cultura política y sentaba las bases de las acciones anticatalanistas llevadas a cabo por la institución, en connivencia con la Diputación Provincial y otras instituciones del franquismo final. La idea de región histórica había derivado en una región política autonómica anticatalana como respuesta a otras ideas nacionalistas y autonomistas. El propio Gobierno Civil lo plasmaba en su memoria de 1977:

El peligro separatista atizado por Cataluña: Preocupante es la posición de los grupos socialistas y democráticos que se limitan como del País Valenciano, ya que han cogido una línea de insistencia en la autonomía, el federalismo, 'las nacionalidades', la Generalitat, la soberanía de los pueblos del Estado español, etc. Obsérvese que han borrado de sus escritos, declaraciones, manifiestos y léxico en general, la palabra España. Para éstos, existen 'las distintas nacionalidades del Estado español', 'los pueblos del Estado español', 'la federación de las nacionalidades' y 'regiones del Estado español'. Nunca España. Esta postura, preocupa a muchas gentes, y puede originar, y de hecho es en determinadas personas, una negación de la unidad de España. [...] Se señala esta actitud, fomentada por intereses catalanes, desde Barcelona, es atizada en el interior por personas con la intención a que nos referimos, se han puesto a hacer el panegírico a todo lo catalán. Sabido es también que se atribuye al Omnium catalán la ayuda con grandes cantidades de dinero, repartidas para 'actividades culturales'. ${ }^{25}$

La motivación claramente respondía a toda una trayectoria política y cultural previa que el alcalde y su equipo municipal dotan de contenido y trascienden el propio discurso de elites para conectar con el sentir de parte de la población local.

25 Ministerio del Interior, Administración Local, Memorias Gobiernos Civiles, Memoria Gobierno Civil de Valencia 1977, 32/11463, Archivo General de la Administración (AGA). 
Lo hizo convencido [Ramón Izquierdo], pero convencido desde el punto de vista que había un sentimiento valencianista que además no coincidía para nada con el sentimiento valencianista catalanista de la Universidad [...] El valencianismo de la universidad se expresaba en valenciano en un idioma similar al que se hablaba en Cataluña [...] Y por tanto yo creo que cuando Miguel Ramón lo hace desde la alcaldía de Valencia lo hace convencido de que se puede reivindicar cosas que no dependan de otros sitios. ${ }^{26}$

Así, se generará un movimiento conservador, populista y regionalista que, tras pasar por UCD-Valencia, tendrá gran éxito político a finales del siglo xx con su integración en el ideario político del Partido Popular de la actual Comunidad Valenciana. Para el caso del personal político proveniente del franquismo será fundamental la potenciación del discurso por parte de unas elites e instituciones locales, integradas en los movimientos e instituciones de la transición, primero, y en partidos políticos, después. Con ello se daba respuesta a unas clases medias tradicionales en un contexto de dislocación social, cultural y de influencia de las teorías nacionalistas periféricas fusterianas. El blaverismo, instrumentalización política del regionalismo "bien entendido" franquista constructor de nación española, ofreció una "respuesta segura" a todos los cambios que se estaban produciendo en los sesenta y setenta desde una cultura política concreta proveniente del regionalismo nacionalcatólico conservador.

\section{A modo de conclusión: elites locales y culturas políticas del nacionalismo español}

Como ha quedado patente a lo largo de estas páginas, el plano local ofrece perfiles muy interesantes para rastrear el desarrollo y difusión de las trayectorias de las culturas políticas del nacionalismo español, especialmente por lo que se refiere a la utilización del discurso regional. La construcción de las mismas en el plano local, así como su cambio y transfor-

26 Entrevista a Héctor Villalba citado en: Vicent Flor, L'anticatalanisme al País Valencià. Identitat i reproducció social del discurs del «Blaverisme» (tesis doctoral, Universitat de València, 2009), $257-358$. 
mación con respecto a ese contacto con la realidad cotidiana o próxima, han sido los aspectos trabajados en el presente trabajo.

Así, a la preponderancia, en el caso de la ciudad de Valencia, de un sector falangista con una concepción de la nación determinada a mediados de los sesenta con tintes regionalistas propios de la tradición política valenciana, podemos encontrar, posteriormente, un refuerzo de la identidad regional propio de la cultura política nacionalcatólica y que será fundamental en la construcción autonómica posterior. Esa cultura política pondrá el acento en el aspecto regional como parte de la pluralidad de la nación, y enfatizará el aspecto político de la misma, alejándose de aspectos folclóricos reivindicados con anterioridad que, aunque también presentes, no serán los únicos reivindicados. En esta última fase, la defensa del anticatalanismo tendrá un peso fundamental en tanto en cuanto definidor de la nueva región política valenciana pegada a la nación española.

Por tanto, un estudio complejo de las culturas políticas nacionalistas en la dictadura nos permite ver su continuidad, permeabilidad, modificación y transferencia en la localidad. El desarrollo de un importante espectro de partidos regionalistas en la década de los ochenta, con gran rédito electoral en muchos casos, será una clara consecuencia de todo lo anterior. La existencia, en la actualidad, de culturas nacionalistas españolas que siguen remitiendo a algunos elementos que hemos comentado es prueba de esa adaptación, perdurabilidad y pervivencia que tienen en lo local las culturas políticas nacionalistas franquistas. Valencia es buen ejemplo de todo lo anterior. 